\title{
Efecto de dos formas de suplementación de vitamina $D$ en el crecimiento y metabolismo óseo del lactante
}

\author{
Edda Lagomarsino F. ' : Pilar Arnaiz G.', \\ Pablo Vial C. '; Felipe Heusser R. ! Marisol Cantwell A.?; \\ María C. Johnson P., Arnaldo Foradon C. ${ }^{4}$ \\ Effects of two forms of vitamin D supplementation \\ on bone metabolism and infants growth
}

\begin{abstract}
The effects of e ther cai y doses of 400 Uا $\forall$ lamin D. along the firsi slx months $\sigma^{\circ}$ life or $600000 \mathrm{~L}$ doses a cges : and 0 month on tore metabolism and growth were evo ucted in two groups of nomal intorits up lo the eighth montr of life. Weigrt, heighr, head eircumference, seruth calc urn, prosphorus, alkcline phosoliatcse, parathyroid

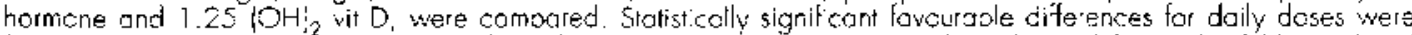
found from tre thire month on heigh: from the sixth month on weicht and fron the eighth month of life on tread c reunference. Blooc pressure wos highe suecially $30,60,90$ and 120 days diter the lirst 600 000 WU vit D megaccse. Alean serum $1.25(\mathrm{OH})$, $\mathrm{D}$, was cors stently higher in saliets given masive vilamin doses, $1110 \mathrm{cnd}$ $96 \mathrm{Pg} / \mathrm{r}$ at third and sixi- mo-th, as compaed with $55 \mathrm{pg} / \mathrm{ml}$ respective $y$ in subjects under caly dosesil. No slaristic other differences were found in otrer of blood measuremen's. Susplementation of 400 daiv IU, is physiclocically scunder as it aromotes growth, avoids risk of intoxication and polentia of marsien! induced

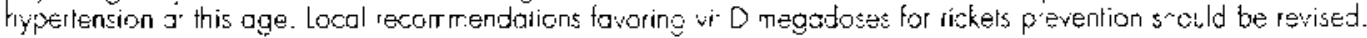

(Key words: bone metcko ism, vitamin D, irfant, growt, arterel rypertension.)

La forma activa de la vitamina $D$ (vit D), la 1-25 dihidroxi vit $\mathrm{D}\left(1,25 \mathrm{OH}_{2}\right.$ vit $\left.\mathrm{D}\right)$, participa como una hormona en la regulación del metabolismo del calcio y fosforo. La cantidad necesaria para alcanzar la función fisiologica óptima cn el lactante depende de numerosos factores como edad, ritmo de crecimiento, absorción intestinal, enfermedades coexistentes y clima ${ }^{3}$.

Los requerimientos de vit $\mathrm{D}$ durante el primer año de vida se estiman entre 100 y 400 UI diarias $^{2}$ ? La leche materna aporta aproximada-

1. Departamento de Pediatría, Faculaad de Medicima, Lniver'sjalad Católica de Chile.

2. Enfermera Universitaria. Centro de Diagnostico, Facultad de Medicina, Universidad Católica de Chile.

3. Bioquímico. Laboratorio de Medicina Vuclear, Facultad de Medicina. Universidad Católica de Chile

4. Laboratorio de Medicina Nuclear, Facultad de Medicina, Universidad Católica de Chilet.

Fuente de Apoyo: Direction de In vestigaciones Uujversidad Católica DIUC $90 / 84$ mente $20 \mathrm{UL} / 1^{4}$. No está claro hasta qué punlo los lactantes de término, sanos, amamantados, requieren complemento de vit $\mathrm{D}$ en los primeros seis meses de vida, ya que la leche humana provee calcio (Ca) y fósforo ( $\mathrm{P}$ ) en una forma más utilizable para la mineralización, sin necesitar grandes cantidades de vit D. Aun así, se recomienda administrar 400 UI * día a estos niños, hasta que el asunto esté totalmente dilucidado?

El Ministerio de Salud de Chile, para prevenir el raquitismo por deficiencia de vil $D$ en menores de un año, ha recomendado para la Región Melropolitana de Santiago un suplemento conocido como "golpe vitamínico" consistente en 600000 UI de vit D, administrado en los meses primero y sexto de vida. El comité de nutrición de la Academia (Norte) Americana de Pediatría $y$ otros aconsejan aportes diarios de 400 UI de vit $\mathrm{D}$ durante el primer año de vida mediante leche curiquecida con la vitamina 0 , en caso de lactantes alimentados al pecho, un 
suplemento acuoso diario, desaconsejando otros alimentos enriquecidos en vitamina $D$, para evitar la intoxicación ${ }^{2}$. 3. 5 . La recomendación local significa una dosis total 20 veces mayor que las recién señaladas. Si bien los informes de morbilidad del ministerio muestran drástica reducción en la incidencia del raquitismo en los ninoos. no se han evaluado las consecuencias de la megadosis en el metabolismo Ca-P, que podrían ser deletéreos en el desarrollo del niño. Para subsanar esta carencia, se diseñó un estudio prospectivo para comparar las dimensiones corporales, presion arterial, calcemia, fosfemia, fosfatasas alcalinas totales (FA), concentraciones plasmáticas de $1.25\left(\mathrm{OH}_{2}\right)$ vit D y hormona paratiroidea (PTH) en lactantes manejados con un régimen de $600000 \mathrm{UI}$ de vit $\mathrm{D}_{3}$ al primer $y$ sexto mes de edad o con 400 UI diarias respectivamente, con el propósito de detectar posibles repercusiones en el metabolismo bseo de unos u otros.

\section{Pacientes y Método}

Se incluycron 78 laclantes en control desde el naciniento en la unidad de pediatría ambulatoria del Cintro de Diagnóstico de la Pontificin Universidad Católica en Santiago de Chile (CEDIUC), nacidos de rérmino, con peso adecuado para la edad gestacional, sin afecciones neonatales, que recibiesen alimentación matema o formulas lácteas de composición y cantidad conocidas y mo ingirieran otras vitaminas además de la vit $\mathrm{D}$ indicada por el equipo. Los participantes fueron asignados por orden de admision a programas altemos: un grupo 1 . de 35 niños, recibió 600 (1) $00 \mathrm{Ul}$ de vit $\mathrm{D}_{3}$ al primer y scxto mes de edad: el gril. po 2. de 43 nin̄os, récibió 400 UI de Vit D al dia (20 gocas) desde el $15^{\circ}$ día hasta complelado el sexto mes de vida. Ambos grupos se incorporaron al programa mensual de control de niño sano del CEDIUC. Se evalub talla, pesso. circunferencia craneana (CC) y presión arterial con efecto Doppler en cada control. A los 15 días, primer, tercer, sexto y octinu mes de vida se hicieron mediciones de calcernia, fostemia, fosfatasas alcalinas, hormona paratitoidea y $1.25(\mathrm{OH})$, vit $\mathrm{D}$ en todos Ios cosos. En el grupo 1 las muestras del primer y sexio mes se tomaron 24 horas después de suministcar las 600000 Ul de vit D por via oral. Para las determinaciones de calcemia, fosfemia y cosfatasas alcalinas se utilizó un autoanalizador Technicon de tercera generación. que además informo glicenia, uremia, colesterol. proteínas totales y albuminemia. La I,25 (OH) vit D se estudió con radicinmiunoanálisis en el Laboratorio de Medicina Nuclear de la Universidad Catolica y la hormona paratiroidea con el conjunto "PTH Kit D SL" (Diagnostic Systems Laboratories), que mide la fracción media de la hormona, que viene en fragmentos de 44 a 68 aminócidos. En los niños del grupo I se empleó la solu. ciśn de vit D para uso oral enyasada en ampollas de $1 \mathrm{ml}$ por el Laboralorio Chile, conteniendo $15 \mathrm{fng}$ de vit $\mathrm{D}_{3} 0$ colecalciferol, equivalentes a 600000 UI. Como en cl comercio no era posible obtener una solución oral que tuvie. se exclusivamente vit $D$ en gotas, el laboratorio mencionado fabricó especialmente para esta investígacion un préparado que efr 20 gotos contenia 400 li de vitamina $D_{3}$ para los níños del grupo 2. La información fue registrada en fichas precodificadas y procesada en un computador digital DEC-10 del Servicio de Ciencias de ta Computación de la Universidad Católica. Para delerminar la significación estadística se aplicaron la prueba del $\chi^{2}$ para las proporciones y la prueba $t$ de Student para los promedios. La inyestigación fue sometida a las regulaciones éticas de la Universidad Católica. Los padres dieron su consentimiento. informado por escrito, antes del ingreso de los nihos al estudio.

\section{Resultados}

En los primeros cuatro mescs de vida el peso y el perímetro craneano aumentaron de manera semcjante en ambos grupos, diferenciándose en el scxto y el octavo mes en favor de los niños del grupo 2 ( $p=0,02$ para ambas medidas). El peso (promedio) a los 8 meses fue de $7908 \mathrm{~g} \mathrm{cn}$ los niños que recibieron $600000 \mathrm{UI}$ y $8712 \mathrm{~g}$ $(\mathrm{p}<0,05$ ) en los del grupo 2. Los promedios del perímetro craneano fueron $44,3 \mathrm{~cm}$ y $45,5 \mathrm{~cm}$ respectivamente. En talla se registró mayor incremento en el grupo 2 desde los tres ineses, para alcanzar una diferencia de $2,7 \mathrm{~cm}$ desde $\mathrm{cl}$ quinto mes ( $p=0,0003$ ), que persistía después del octavo mes $(67,5 \mathrm{~cm}$ en el grupo 1 y 70,5 en el grupo 2, figura 1$)$. En las dos series de niños las dimensiones corporales estaban, sin embargo, dentro de una DE de los estándares.

La presión arterial era similar en ambas seties antes de iniciar la ingestión de vit $D$. Después fue sostenidamente mayor en el grupo 1 , existiendo diferencias significativas entre los promedios $(\mathrm{p}=0,0002)$ y por mes de control ( $p=0,0014)$, especialmente en los meses segundo, tercero y cuarto. Las cifras de ambos grupos estuvieron dentro del percentil 95 para la edad, pero las del grupo I sobrepasaron el percentil 90 para la edad (figura 2 ).

No se encontraron diferencias significativas en las concentraciones plasmáticas de $\mathrm{Ca}, \mathrm{P}$. fosfatasas alcalinas, glucosa, nitrógeno ureico, colesterol, proteínas totales, albúmina. Las concentraciones séricas de $\mathrm{Ca}, \mathrm{P}$ y fosfatasa alcalina variaron en el transcurso de los meses, pero siempre se mantuvieron dentro de límites normales para la edad (figura 3 ). 


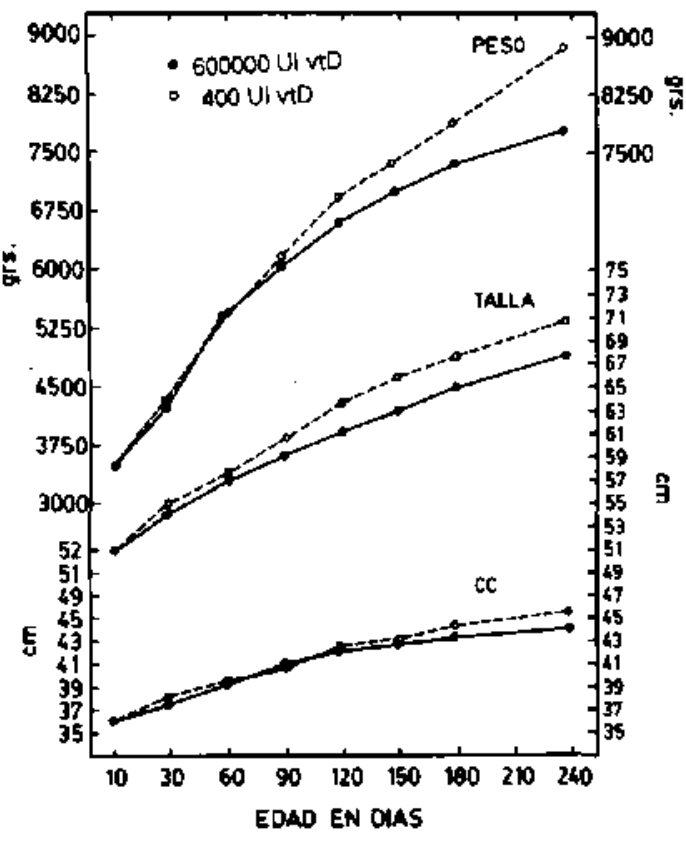

Figura 1: Peso, talla y circunferencia craneana (CC) en lactantes que recibieron vitamina D $600000 \mathrm{Ul}(\bullet)$ al primer y sexto mes y 400 LI(o) diarias, entre los 15 y 240 dfas de vida.

Las concentraciones plasmáticas (promedios) de $1,25(\mathrm{OH})_{2}$ vit $\mathrm{D}$ en el grupo I fueron $110 \mathrm{y}$ $96 \mathrm{pg} / \mathrm{ml}$ el primero y sexto mes de vida, res- pectivamente, y fluctuaron entre 50 y $60 \mathrm{pg} / \mathrm{ml}$ en el grupo 2 (figura 4).

\section{Comentario}

La concepción tradicional de la relación entre vit $\mathrm{D}$ y $\mathrm{Ca}$ es demasiado limitada y ha sido anpliamente superad $a^{6,7}$. Se ha demostrado receptores $1-25(\mathrm{OH})$, vit $\mathrm{D}$, en variados tejidos, por lo que se considera que la vit $\mathrm{D}_{3}$ es una activadora y moduladora somatotrófica general. Además de su conocida acción a nivel del intestino, riñón y hueso, actúa también en el tejido nervioso, endocrino y brganos efectores como mamas y útero, influyendo en la regulación endocrina de todas las hormonas, en la innervación muscular, en la respuesta al estrés, en funciones inmunitarias, fertilidad, embarazo y lactancia ${ }^{8.9}$.

El exceso de vit D puede ser dañino. La intoxicación se expresa en hipercalcemia, alteraciones de la mineralización ósea y aumento del producto $\mathrm{Ca}-\mathrm{P}^{7}$. Sus manifestaciones se confunden con otras afecciones frecuentes en la infancia: poliuria, vómitos, hipertensión arterial, detención del crecimiento². En Chile se administra vit $\mathrm{D}_{2} \circ \mathrm{D}_{\hat{\mathfrak{n}}}$. que tienen una vida media fisiológica de aproximadamente un mes, en dosis

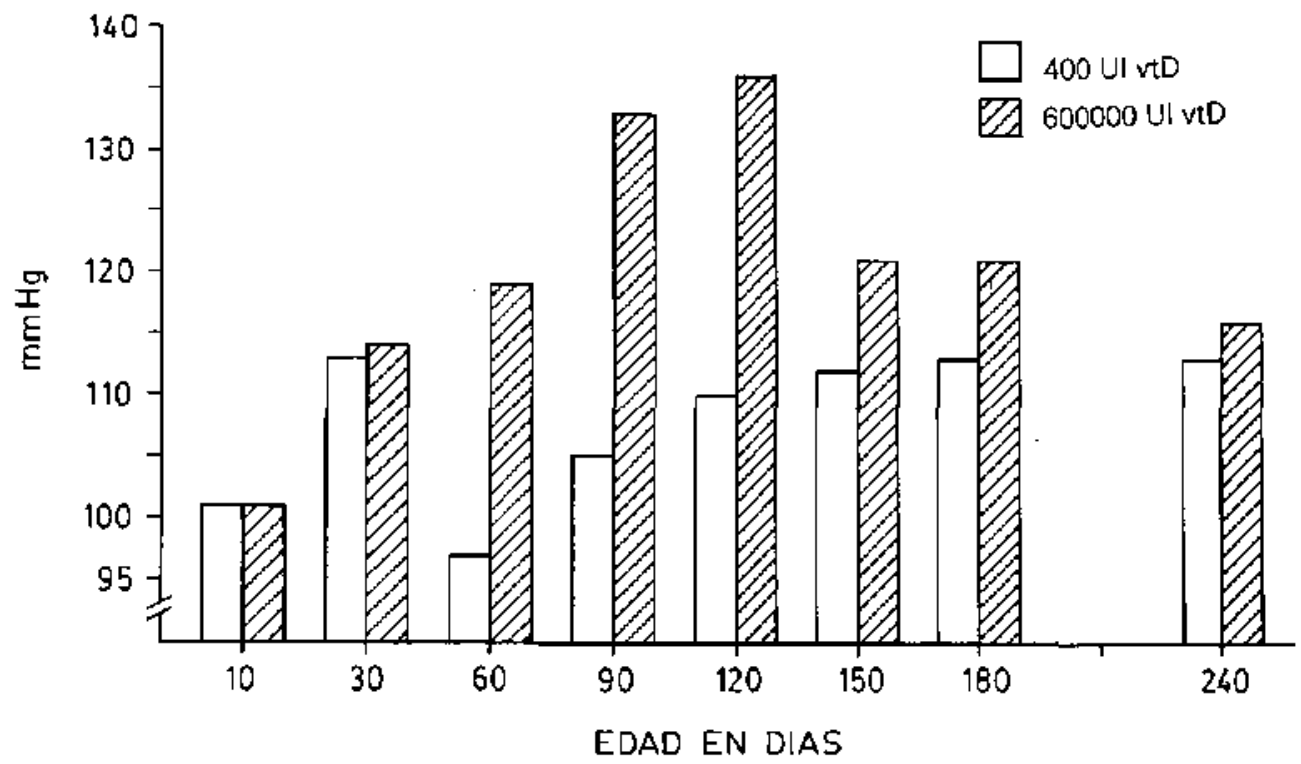

Figura 2: Presión arterial en lactantes que recibieron vitamina D $600000 \mathrm{UJ}$ al primer $y$ sexto mes y 400 UI diarias entre los 15 y 240 días de vida. 

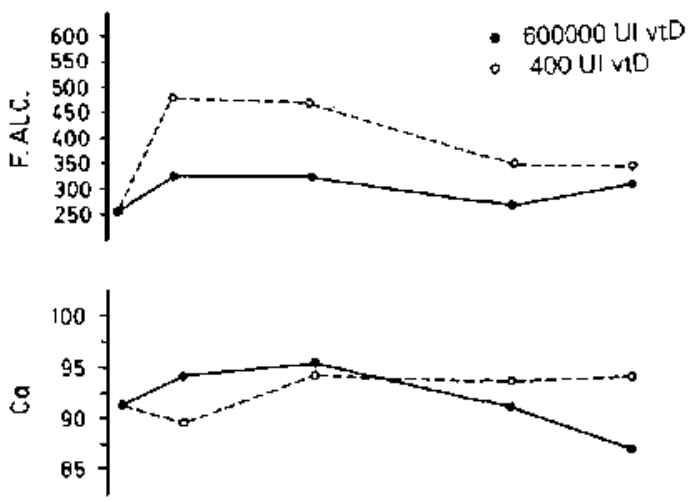

a

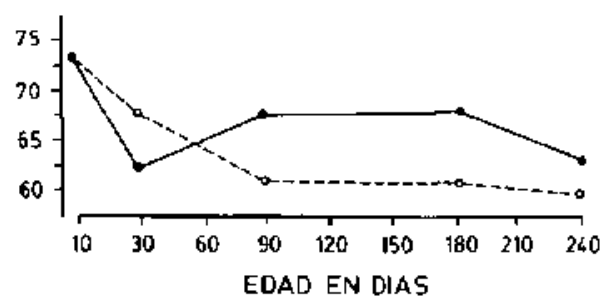

Figura 3: Concentraciones plasmáticas de fósforo (P). calcio (Ca) y fosfatisas alcalinas $(F$ A.tc) en lactantes que recibian vitamina $D 600000 \mathrm{Ul}(\cdot)$ al primero $y$ sexto mes y 400 UE (o) diarias entre los 15 y 240 días de vida.

de 600000 UI al primer y sexto mes de vida. Puesto que la vitamina se almacena en el organismo, esta práctica puede ocasionar intoxicaciones, de las que ha habido reportes ${ }^{10-12}$. Esta forma peculiar de administración se originó en la alla tasa de raquitismo que se tegistraba hace algunos años en Chile: $24 \%$ de los menores de 4 años en 1931 y $38,3 \%$ entre los niños hospitalizados $^{17-14}$. Si bien con suplementos más fisiologicos de vit $D$ se obtiene igual efecto antirraquitjeo ${ }^{8}$, el buen éxito de la antigua recomendación ha becho renuentes a las autoridades de salud a cambiarla, posiblemente por desconfranza en el cumplimiento del esquema de administración oral diaria, prefiriéndose otro controlado. En nuestra experiencia no hubo dificultades para que las madres de estratos económicos medin y medio bajo entregasen a sus hijos el aporte diario de vit $\Gamma$ que requerían, por lo que pensamos que la desconfianza no se justifica actualmente. Además avalan esta creencia la actitud favorable de la comunidad nacional ante campañas de salud pública como inmunizaciones y preven- ción del cólera y una cxperiencia de 8 años en el CEDIUC, en que hemos indicado a todos los lactantes bajo control dosis diarias de $400 \mathrm{UI}$ sin haber registrado casos de raquitismo por falta de vit D.

El aumento significativo del metabolito activo de la vit $\mathrm{D}$ en el plasma al día siguiente de la administración de una megadosis de vit $D$ no se tradujo en variaciones simultáneas en el plasma de $\mathrm{Ca}, \mathrm{P}$. hormona paratiroidea y fosfatasas alcalinas, esta última una isoenzima osea que cxpresa indirectamente actividad osteoblástica. Sin embargo se registró aumento de la presión arterial, que podría deberse a mayor dismonibilidad de Ca en las paredes de los vasos sanguíneos ${ }^{15}$. Existe una correlación positiva entre la concentración de Ca plasmático y la presión arterial ${ }^{16}$, pero el rol de la vit $D$, al respecto, no ha sido aclarado, aunque se han descrito elevaciones transitorias de la presión arterial con dosis terapéuticas de vit $\mathrm{D}^{17,18}$. Es posible que un estudio prospectivo de seguimiento poblacional permitiese dilucidar el efecto que con el tiempo pudiese tener esta hipertensión relativa.

El mayor crecimiento asociado a dosis pequeñas diarias de vitamina $D$ sugiere que esta forma de administración tiene ventajas claras de tipo fisiológico sobre las megadosis, en coincidencia con publicaciones de hace treinta años ${ }^{19}$. 20. El control a Iargo plazo de estos njños sería útil

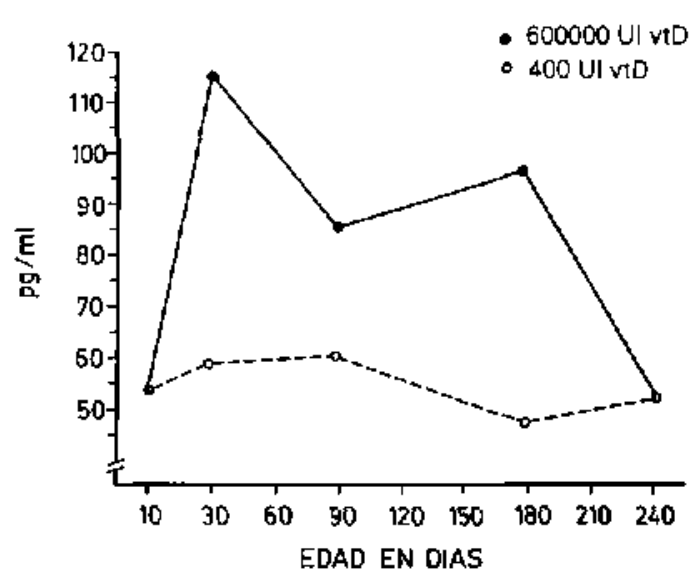

Figura 4: Concentraciones plasmáticas de 1,25 dihidroxi vitamina $D$ en lactantes que recibieron 600000 UI (0) al primer $y$ sexto mes y $400 \mathrm{UI}(0)$ diarias de vilamina $D$ entre los 15 y 240 días de vida. 
para determinar si estas diferencias persisten. Con dosis orales de 400 UI de vit D suministradas a lactantes alimentados con leche materna - leche de vaca modificada y enriquecida con vit $D$ se ha logrado erradicar totalmente el raquitismo ${ }^{3-5}$.

Parece llegado el momento de revisar la antigua norma ministerial, ya que un suplemento de 400 UI de vit D parece ser fisiológicamente más adecuado que en megadosis de $600000 \mathrm{UI}$ dos veces al año, pues los indices de crecimiento son mejores con el primer esquema y los "golpes vitamínicos" representan riesgos documentados de intoxicación y potenciales, no bien calculados aún. sobre la presión arterial.

\section{Resumen}

Se evalú́ el efecto sobre el metabolismo óseo y crecimiento del lactante menor de suplementos de vitamina D de $600000 \mathrm{UI}$ en cl primero y sexto mes (norma ministerial) ante una dosis diaria de 400 UI hasta el sexto mes, en dos grupos de lactantes normales. En controles mensuales se midieron peso, talla, circunferencia craneana, presión artcrial, calccmia, fosfemia, fosfatasas alcalinas, hormona paratiroidea y $1.25(\mathrm{OH})_{2}$ vit $\mathrm{D}$. Se encontraron diferencias estadísticamente significativas en favor de los niños que recibían 400 UI diariamente, a contar del tercer mes en la talla, del sexto mes en e] peșo y del octavo mes en la circunferencia craneana. La presión arterial fue más alta en los pacientes que recibieron megadosis semestrales de la vitamina, especialmente $30.60,90$ y 120 días después de la primera de cllas. La concentración plasmática media de $1,25(\mathrm{OH})_{2}$ vit $\mathrm{D}$ fue más alta en los casos tratados con megadosis ( 110 y $96 \mathrm{pg} / \mathrm{ml}$ en el primero y e] sexto mes respectivamente). que en los que recibía dosis diarias $(55 \mathrm{pg} / \mathrm{ml}$ en el primero y sexto mes). No se encontraron diferencias en las restantes variables sanguíneas analizadas. La administración de 400 UI diarias es más fisiológica, promueve mayor crecimiento, evita riesgos de intoxicación a corto plazo y potencial a largo plazo por la hipertensión inducida a esta edad. La norma ministerial debiera ser revisada.

(Palabras clave: metabolismo óseo, vit D, crecimiento lactantes, hipertension arterial.)

\section{Referencias}

1. Lichtestrtein P. Speaker L. Tsang RC. Mimouni $F$. Gormley $C$ : Calcium-regulating hormones and minerals from birth to 18 months of age: a cross sectional study. Effects of sex, race, age, season and diet on vitamin D status. Pediatrics 1986; 6: 883-890.

2. American Academy of Pediatrics. Committee on $\mathrm{Nu}$ trition: The prophylactic requirement and the toxicity of vitatnin D. Pediatrics 1963:33:512-525.

3. Anerican Academy of Pediatrics. Committee on Matrition: Vilamin and mineral supplement needs in normal children in the United States. Pediatrics 1980; 66: $1015-1021$

4. Greer F, Hotlis B, Cripps $D, T$ satng $R$ : Effects of matemal ultraviolet $B$ irradiation on vitamin $D$ content of human milk. J Pediatr 1984; 3: 43I-433.

5. Bransby ER, Berry W, Taylor D: Study of vitamin D intakes of infasts in 1960. Brit Med J 1964; 1: 166!-1663.

6. De Luca HF, Schnoes HK: Vitamin D: Recent anvances. Ann Rev Biochem 1983; 52: 411-439.

7. Stumpf WE: Viramin D soltriol the heliogenic steroid hormone: somatotropic activator and modulator. Discoveries from histochemical studies lead to new concepts. Histochem 1988: 89: 209.

8. Sperker BL. Trang RC: Vitamin D in infancy and childhood: factors delennining vitarnin $\mathbf{D}$ status. Adv Pediatr 1986; 33: 1-22.

9. Specker BL. Lichtenstein P. Mimouni F, Gumley C. Tsang $R C$ : Calcium-regulatiog hormones and minerals from birth to IB month of age: a cross sectional study. Il efects of sex, rece, age season and diet on serum minerals parathyroid hormone and calcitonin. Pedialrics 1986: 77: 891.896.

10. Patterson C: Vitamin D poisoning. Survey of causes in 21 patients with hypercalcemia. Lancet 1980: 31: 1164- 1167.

I1. Molina $H$, Mena P, Vial P. Ferrainder, E, Alcázar $M$, Muzze $S$ : Intoxicación por vitamina $D$ en el lactante. Rev Chil Pediatr 1984: 55: 270-273.

12. Americtin Academy of Pediatrics. Commitlec on Nutrifion: Vitamin D intake and the hypercalcemia syndrone. Pediatrics 1965; 35: 1002-1023.

13. Baeza-Goñi A: Raquitismo, su frecuencia en Santiago de Chile. Rev Chil Pediatr 1931: 2: 14I-146.

14. Schwarzemberg J: Profilaxis del raquitisno por medio del golpe vitamínico. Rev Chil Pediatr 1942: 13: 502. 615.

15. Karinja B. McCarom $D$ : Calcium and hipertension. Ann Rev Nutr 1986; 6: 475-494.

16. Earl JH. Kurizman NA, Moser RH: Hypercalcemia aud hypertension. Ann Intern Med 1966: 64: 378-381.

17. Sowers $M F$, Wallace $R B$, Lemke $J H$ : The association of intakes of vit D and calcium with blood pressure among women. Am J Clin Nutr 1985; 42: 135-142.

18. Linden $V$ : Vitamin $\mathrm{D}$ and myocardila infarction. Br Med J 1974; 3: 647-650.

19. Stestrms G. Jeans PC, Wandecas V: The effect of vitamin D on lineas growth in infancy. J Pedjatr 1936; 9 : $14-16$.

20. Sfycer F, Poole H, Cootey $T$. Macy $J$ : Relationship beiween vitamin $D$ intakes and linear growth in infants. Proc Soc Exp Biol Med 1937; 37; 499-502. 